\title{
El modelo de Contingencia de Fiedler en Procesos de Fabricación Progresiva
}

\author{
MIGUEL BARÓN \\ Universidad de Sevilla

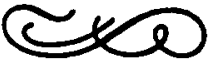

\section{Resumen}

En las organizaciones productivas, la influencia de los líderes se manifiesta en el nivel de eficacia grupal y como consecuencia en el logro de objetivos. Comprobar la capacidad de predicción de los modelos sobre el liderazgo existentes en el marco teórico de la Psicología Social puede resultar beneficioso para el diseño de futuras organizaciones y la remodelación de las actuales. Estudiamos aquí el Modelo de Contingencia de Fiedler, aplicándolo a procesos de fabricación progresiva. Los resultados obtenidos nos llevan a aceptar las bipóteis del modelo en solamente tres de las ocho condiciones de favorabilidad que éste plantea. Con la mísma valoración del tipo de liderazgo del modelo nos muestran también los datos:

1. Que no existe correlación entre el rendimiento de los grupos y el grado de experiencia de los lideres.

2. La falta de correlación entre el nivel de accidentabilidad grupal y los diferentes tipos de liderazgo.

3. Un rendimiento superior en aquellos grupos en los que el tipo de motivación del lider $y$ de los superiores de éste coinciden.

\section{Abstract}

In productive organizations, the influence of the leader is shown at the level of group efficiency and as a result in the achievement of objectives. Verifying the prediction capacity of the existing leadership models, within the theoretical framework of Social Psycholoby, could bave beneficial results for the design of future organizations and the remodelling of existing ones. Here the Fiedler Contingency Model is studiend by applying it to progressive manufacturing processes. The results abtained allow us to accept the bypotheses of the model in only three of the eight favourable conditions that are suggested. With the same assessment of the type of leadership of the model, the following data is also shown:

1. There is no correlation between group production and the level of experience of the leaders.

2. There is an absence of correlation between the range of group accident variation and the different types of leadership.

3. There is higher production in those groups in which type of leader motivation coincides with bis superiors'.

Dirección del autor: Facultad de Psicología, Universidad de Sevilla. Aptdo. 3128. 41080 Sevilla. 


\section{INTRODUCCION}

El liderazgo es uno de los procesos grupales básicos de forma que el fenómeno grupal sería totalmente incomprensible si prescindiéramos de él (Morales, 1985).

Básicamente, el liderazgo significa poder sobre otras personas y el poder sobre otros permite a un hombre hacer cosas, obtener objetivos y llevar a cabo acciones que serían inalcanzables por él mismo. Sin embargo, el poder sobre los demás también suele implicar cierto grado de consentimiento, o al menos, cierta sumisión aceptada, por la que el líder y su organización tienen que pagar un precio (Fiedler, 1971).

En las organizaciones, compuestas por personas que tratan de conseguir fines comunes establecidos explícitamente por medio de esfuerzos, habilidades, energía y otros elementos importantes, al tiempo que desempenan distintos roles en función de la estructura que en ellas se desarrolla (Peiró, 1984), el conocimiento y la comprensión científica del liderazgo es un tema de vital importancia para la consecución del éxito y de los objetivos propuestos.

Existe un considerable acuerdo, entre los estudiosos del tema, en que el éxito o el fracaso de cualquier organización depende, en buena medida, de la calidad de sus líderes. Schultz (1978) señala que aproximadamente la mitad de todas las nuevas industrias fracasan y tan sólo un tercio superan los cinco años, atribuyendo, en la mayoria de los casos, la causa del fracaso a un liderazgo defectuoso. Miljus (1970), indica que todas las consideraciones que afectan al futuro éxito de una industria, sitúan al liderazgo como un factor crítico que podría llegar a convertirse en el único.

Las organizaciones dedicadas a la fabricación progresiva (trabajo en serie) no son una excepción a lo expuesto. Entendiendo a éstas como sistemas abiertos (Katz y Kahn, 1966), quedan definidas como un complejo de unidades inter-relacionadas, en donde la especificación jerárquica de su estructura y el adecuado funcionamiento de sus partes, resultan de una importancia crucial.

El presente trabajo ha tratado de estudiar el liderazgo en procesos de fabricación progresiva por varias razones:

- Porque afecta a la vida cotidiana de miles de personas y gran número de organizaciones.

- Porque puede resultar de interés, que un tema de importancia tradicional en la Psicología social, como el del liderazgo, sea analizado en un contexto industrial que se encuentra en un continuo proceso de adaptación al medio y sus demandas (nuevas tecnologías, robótica, informática, etc.).

- Porque puede aportar alguna luz en la toma de decisiones para políticas futuras de este tipo de organizaciones.

Hemos tomado para ello el Modelo de Contingencia de Fred E. Fiedler (1965-1967) que fue realmente la primera y principal teoría que, especificamente propuso relaciones de contingencia en el campo del liderazgo. Su utilidad se sitúa sobre todo en el campo de la Psicología de las organizaciones tratando de solucionar gran parte de las incógnitas que hasta entonces se planteaban en el estudio y conocimiento del liderazgo eficaz (Peiró, 1984). 
El Modelo de Contingencia goza de un extraordinario compendio de datos experimentales que empezaron a publicarse a partir de 1953, representando más de 20 años de investigaciones en más de 1.600 grupos de laboratorio o naturales; produciendo más de 300 estudios que han tratado de apoyar, desestimar o precisar algunos aspectos de las originales proposiciones (Rubio, 1986).

Entre las revisiones realizadas al modelo, podemos destacar las realizadas por Mitchell (1970), Fiedler (1971), Rice y Chemers (1973) y Fiedler (1978) que se decantan a favor de que las hipótesis del modelo tienen un sólido soporte empírico y las realizadas por Graen (1970), Ashour (1973), Hosking (1978) y Rubio (1986) que niegan dichas aportaciones empíricas a la teoría.

Se asienta la teoría en la idea de que el rendimiento en la consecución de objetivos, la eficacia del grupo, es contingente a la relación que se produce entre el estilo de liderazgo y el grado en que la situación grupal permite al líder poner en práctica su influencia.

No aporta el modelo tipologías de rasgos de personalidad o constructos hipotéticos situacionales que de forma anticipada garanticen la eficacia de un determinado líder. El líder sólo tendrá éxito, según Fiedler, si además de reunir las cualidades personales necesarias, coinciden éstas en la situación social propicia para que se produzca su máxima influencia en el grupo y en la obtención de los objetivos de éste.

Como ya hemos mencionado, la presente investigación se desarrolla en una organización de fabricación progresiva. La expresión «trabajo en serie» (trabajo en cadena), tiene su origen en el francés trabail a la chaine, ciertamente cargado de connotaciones peyorativas; en EE.UU. se le denomina mass production que cuando quiere especificarse cambia por progressive manufacture o bien conveyor work, denominándose al mismo tiempo a las personas que trabajan en las líneas de fabricación como the man in the line. En alemán se usan las expresiones Fliessarbeit (trabajo fluyente) y Laufenden band (cinta continua); es por renunciar a la imagen del trabajador encadenado por lo que usamos del término "fabricación progresiva" para referirnos a este tipo de trabajo (Siguan, 1963).

En la fabricación progresiva, el total del trabajo se divide en múltiples operaciones simples, de manera que cada puesto de trabajo añade una operación al proceso, con lo que al final de la línea el producto queda totalmente terminado y preparado para su inmediato uso. El proceso se complica si tenemos en cuenta que la mayoría de las líneas de fabricación no hacen más que producir parte del conjunto, estableciéndose de esta forma líneas primarias, secundarias, etc. Ello nos da idea de un soporte organizacional bastante complejo, funcionando en todas sus partes como la maquinaria de un reloj de precisión, con unos ritmos exactos, obedeciendo a unos cálculos previos exhaustivos.

La mayor parte del personal de este tipo de procesos queda constituido por obreros especializados en las líneas de producción y una minoría de profesionales cualificados. Son frecuentes y continuos los conflictos interindividuales debido al alto nivel de inter-relaciones necesarias para la fabricación del producto (Leplat, 1977).

Los conocimientos actuales en el campo del liderazgo, más en concreto 


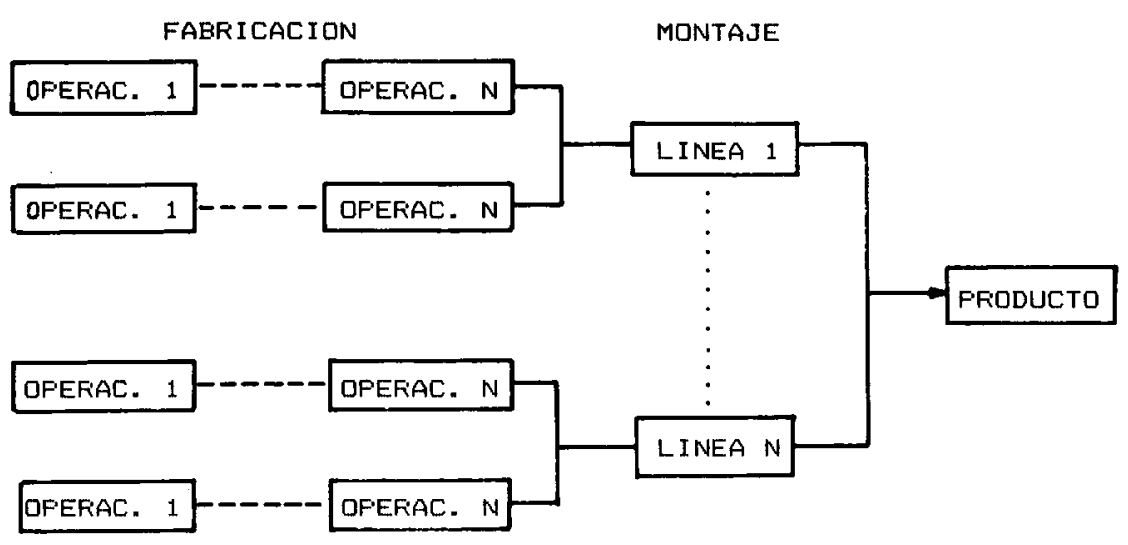

Esquema del proceso de fabricación progresiva

sobre el Modelo de Contingencia de Fiedler, así como el terreno de aplicación del presente estudio, nos ha llevado a la proposición de dos tipos de hipótesis:

\section{a) Tipo $I$}

Nos interesa, en primer lugar, confirmar la influencia de las variables independientes que presenta el modelo estudiado, en el rendimiento y la consecución de los objetivos grupales en procesos de fabricación, adecuándonos a los tres tipos de labores más diferenciadas y significativas que estos procesos presentan:

- Fabricación progresiva (automatizada y semiautomatizada).

- Labores de mantenimiento.

- Labores de montaje.

Estas hipótesis, que denominamos de «Tipo I» son las siguientes:

1. En un grupo de trabajo, caracterizado por tener una alta favorabilidad situacional para que el líder pueda ejercer su influencia, son más eficaces los líderes motivados por la tarea que los líderes motivados por las relaciones interpersonales.

2. En un grupo de trabajo caracterizado por tener unas intermedias o regulares condiciones de favorabilidad situacional para que el líder pueda ejercer su influencia, son más eficaces los líderes motivados por las relaciones interpersonales que los motivados por la tarea.

3. En un grupo de trabajo caracterizado por tener muy baja favorabilidad situacional para que el líder pueda ejercer su influencia, son más eficaces los líderes motivados por la tarea que los motivados por las relaciones interpersonales.

b) Tipo II

En este segundo tipo de hipótesis hemos pretendido estudiar la relación entre cierto tipo de factores propios de la fabricación progresiva (y de sus 
esquemas organizacionales) y la efectividad del ejercicio del liderazgo. Estos factores quedarán centrados en:

- Experiencia del líder, entendiendo ésta como número de años en el desempeño del puesto.

- Actitud y motivación de los superiores jerárquicos del lider.

- Nivel y grado de accidentabilidad grupal.

Las hipótesis de este "Tipo II" las exponemos como sigue:

1. El grado de experiencia del líder influye de forma significativa en el nivel de eficacia grupal.

2. Cuando el tipo de motivación de los superiores del líder coincide con el tipo de motivación del líder, el rendimiento del grupo es mayor que si estas motivaciones son diferentes.

3. Tanto el nivel como el grado de accidentabilidad son mayores en aquellos grupos cuyo líder se encuentra motivado por la tarea.

\section{METODO}

\section{Muestra utilizada}

El número de sujetos participantes en este estudio ha sido de 515 (todos varones) de los cuales 495 han sido operarios especialistas y profesionales especializados, 14 han sido considerados como lideres, siendo todos ellos «mandos intermedios» y 6 lo han sido como superiores jerárquicos de los líderes estudiados. La maestra ha sido seleccionada partiendo de una muestra más amplia de 1.570 sujetos, atendiendo principalmente a dos tipos de criterios:

- En orden al tipo de trabajo realizado en el conjunto de la organización, teniendo en cuenta que los grupos se ajustaran el máximo posible a la puesta en práctica del estudio de las hipótesis del Modelo de Contingencia así como a una mayor generalización de los resultados (grupos más numerosos y menos estructurados).

- Por necesidad de invalidación de cuestionarios de LPC debido a la obtención de una puntuación intermedia en la escala.

\section{Diseño}

Hemos utilizado para el estudio un diseño factorial múltiple de tres factores, sobre grupos estáticos, del tipo $4 \times 2 \times 2$ con tres variables independientes, las cuales dada la imposibilidad de manipulación, han tenido que ser estudiadas como variables asignadas. Estas variables han sido:

a) Favorabilidad situacional: grado de favorabilidad de un grupo según el cual, el lider puede ejercer su influencia o control sobre la conducta de los demás miembros del grupo.

Resultan en esta variable cuatro niveles fruto de combinar entre sí las dos dimensiones que define el Modelo de Contingencia: relación lídermiembros y posición de poder del líder, en sus dos grados alto y bajo. 


\section{4}

b) Estructuración de la tarea

Esta dimensión queda determinada también por dos niveles, baja y alta estructuración. Su tratamiento no queda basado en la manipulación experimental, sino en una asignación a los dos niveles, alto o bajo, despues de estudiar los diferentes tipos de trabajos realizados por los grupos; esta asignación se realizó en base a los siguientes parámetros:

- Exigencias de calidad, en lo referente a las tolerancias de fabricación del producto elaborado.

- Medios a utilizar para la fabricación de dicho producto a nivel de máquinas, herramientas, útiles de medición, etc.

- Cantidad de trabajo por unidad de tiempo.

- Grado de manipulación, por parte del sujeto que ocupa el puesto de trabajo, para el cumplimiento de la tarea encomendada (nivel de automatización).

- Nivel de toma de decisiones tanto del operario como del líder en la puesta en práctica del trabajo y en la realización satisfactoria del mismo.

Así pues hemos considerado una tarea como:

- Muy estructurada, cuando en ella existe un elevado nivel de automatización para la realización del trabajo y los sujetos del grupo, tanto líder como demás miembros, tienen una capacidad de decisión muy reducida.

- Poco estructurada, cuando en la realización de la tarea el nivel de automatización es reducido y la cantidad de toma de decisiones bastante alta.

c) Tipo de motivación del lider, donde delimitamos dos niveles:

1. Lider motivado por las relaciones interpersonales, que sería aquella persona que encuentra su mayor satisfacción dentro del grupo cuando sus relaciones con los demás miembros tienen éxito (en la escala LPC con una puntuación total superior a 55 puntos).

2. Lider motivado por la tarea, por el que entenderíamos a aquella persona que obtiene su mayor satisfacción en el grupo cuando éste tiene éxito en la consecución de los objetivos grupales, en la realización eficaz de la tarea (con los resultados de la misma escala LPC su puntuación ha de ser inferior a 45 puntos para ser encuadrado en este nivel).

Son en total ocho las condiciones de las dos primeras variables independientes que, combinadas con los dos tipos de líder, centrado en la tarea y centrado en las relaciones, nos proporcionan un total de dieciséis condiciones experimentales diferentes de las que solamente han podido ser estudiadas 14 al no encontrar grupos que se adaptarán a las condiciones de las tres variables independientes para el octante 6 del Modelo, han sido por tanto 7 los grupos estudiados cada uno de ellos con los dos tipos de líder.

Para las hipótesis relacionadas con el modelo del «Tipo I» la variable dependiente estudiada ha sido el rendimiento del grupo en cada caso. Hemos definido el rendimiento por medio de la incorporación del término productividad, más adecuado para estos tipos de trabajo, siendo entendido como:

"Cantidad de trabajo efectuado por el grupo, en razón del número de horas de presencia efectiva».

Dicha productividad se ha evaluado por medio del trabajo ejecutado 
por aquellos miembros del grupo subordinados a los líderes o mandos en cuestión, siguiendo los tres casos siguientes:

a) Trabajo en serie:

- Procesos automatizados.

- Procesos semiautomatizados.

En los dos casos se ha usado la fórmula:

$$
\mathrm{P}=\frac{\text { Piezas producidas (reales) }}{\text { Piezas } / \text { hora }(\text { gama }) \times \text { horas presencia }} \times 100
$$

b) Trabajo de montaje: Con la misma fórmula anterior.

c) Trabajo de mantenimiento:

Las peculiaridades de este tipo de trabajo han exigido cambiar la medida de la productividad, usando para ello la siguiente fórmula:

$$
\mathrm{P}^{\prime}=\text { Horas de presencia }
$$

El segundo grupo de hipótesis "Tipo II» ha exigido además del uso de la productividad como variable dependiente, de la incorporación del I.F. (Indice de Frecuencia) y del I.G. (Indice de Gravedad) para la medición de los accidentes siendo medidos éstos según las normas de la O.I.T.:

$$
\begin{gathered}
\text { I.F. }=\frac{\text { N. Accidentes con baja laboral }}{\text { Horas trabajadas }} \times 1.000 .000 \\
\text { I.G. }=\frac{\text { Jornadas perdidas }}{\text { Horas trabajadas }} \times 1.000
\end{gathered}
$$

\section{Instrumentos}

Los principales instrumentos de medida y de recogida de datos usados para el estudio que describimos han sido los siguientes:

- Escala LPC de Fiedler (1967), para determinar el tipo de motivación o estilo de dirección de los líderes.

- Escalas de Hunt (1967), para medir la atmósfera del grupo y la posición de poder del líder.

- Test sociométrico de Moreno, usado para determinar el tipo de relaciones líder-miembros, del grupo.

- Gamas de trabajo de cada operación, para determinar el tipo de trabajo asi como para cifrar el cien por cien del rendimiento exigido en cada puesto.

- Archivos de personal, de seguridad e bigiene y de producción, estos últimos centralizados en un ordenador IBM Sistema/7 C3 BRO742A, para determinar datos personales, número y gravedad de los accidentes y rendimiento real obtenido por los diferentes grupos de trabajo. 


\section{Procedimiento}

Es necesario destacar que todos los sujetos participantes en esta investigación, asi como los medios de producción lo han sido en la empresa F.A.S.A. Renault (Sevilla), siendo ésta una organización productiva de fabricación progresiva dedicada a la obtención de cajas de velocidades para los vehículos automóviles de la misma marca.

Ayudados de la jefatura de personal se procedía a informar en primer lugar a los jefes de fabricación de cada área de trabajo sobre la realización de la investigación y los objetivos de la misma. Posteriormente se pasaba a entrevistar a cada uno de los jefes de taller a los cuales a su vez se les pedía que rellenasen las mismas escalas y cuestionarios que después cumplimentarían los líderes de cada grupo. El siguiente paso consistía en realizar la misma operación con los mandos intermedios (líderes) para entrevistar por último a los operarios a las órdenes de éstos.

Paralelamente al curso de las entrevistas se iban tomando los datos acumulados de producción, absentismo, accidentabilidad, etc.

Hay que puntualizar que ni los líderes participantes, ni los operarios o miembros de los grupos conocían con exactitud los objetivos perseguidos por la investigación y que las entrevistas y recogida de datos fueron siempre realizadas por personal perteneciente a la organización y nunca por alguien ajeno a la misma.

\section{RESULTADOS Y ANALISIS}

Para el primer grupo de hipótesis (Tipo I), los resultados obtenidos en cada una de las siete condiciones de favorabilidad situacional estudiadas quedan reflejados en la figura 2, donde observamos el rendimiento medio global obtenido por cada uno de los siete grupos, para los dos tipos de liderazgo. La tabla I resume los distintos ANOVAS realizados con los datos de rendimiento.

\section{FIGURA 2}

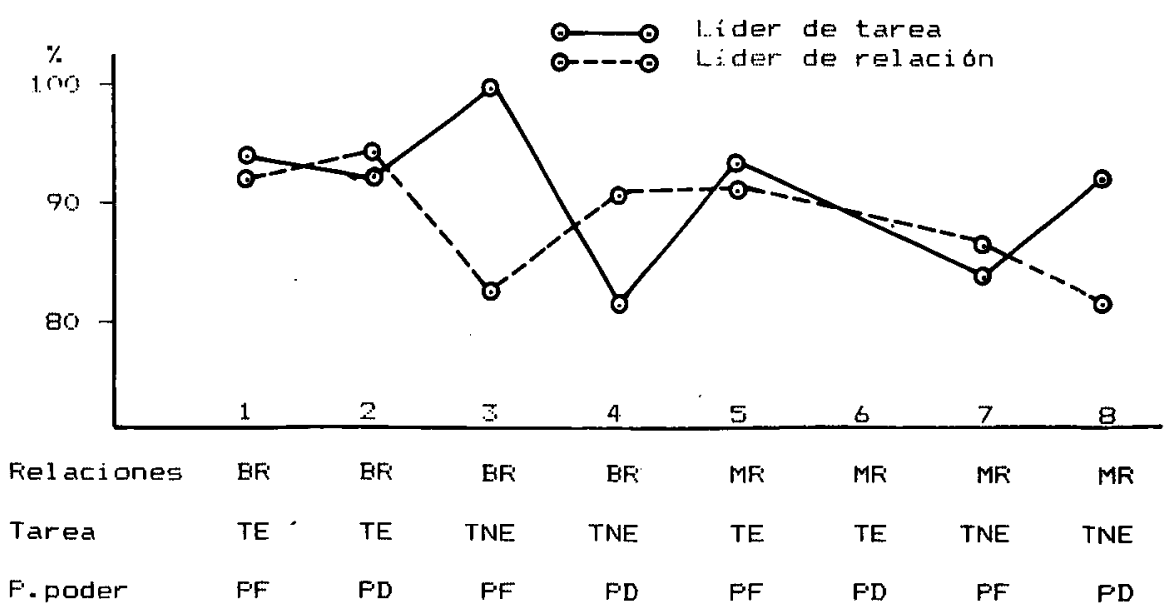


TABLA I

ANOVAS de las distintas condiciones de favorabilidad

\begin{tabular}{ccrrrr}
\hline Situac. & Grupos & Total SS & Trtmt. SS & \multicolumn{1}{c}{ Error SS } & \multicolumn{1}{c}{ F } \\
\hline 1 & $1-9$ & 32.924 & .234 & 32.695 & $5.73 \mathrm{E}-02$ \\
2 & $2-10$ & 14.609 & .250 & 14.359 & .139 \\
$* 3$ & $3-11$ & 526.953 & 404.492 & 122.440 & 26.424 \\
$* 4$ & $4-12$ & 244.929 & 143789 & 101.140 & 11.373 \\
5 & $5-13$ & 29.617 & .054 & 29.562 & $1.47 \mathrm{E}-02$ \\
6 & $6-14$ & - & - & 53. & -7.796 \\
7 & $7-15$ & 59.761 & 5.875 & 53.796 & .873 \\
$* 8$ & $8-16$ & 266.789 & 182.836 & 83.953 & 17.423 \\
\hline
\end{tabular}

- Significativamente diferentes.

En las condiciones I, II y III, de alta favorabilidad situacional, relacionadas con la primera hipótesis planteada, los datos nos llevan a rechazar la bipótesis para las dos primeras situaciones y aceptarla para la situación III.

La segunda hipótesis se contempla en las situaciones IV, V, VI y VII del Modelo; como podemos observar en la tabla I, se acepta solamente en la situación IV. Para las condiciones V, VI y VII no se ha podido confirmar; en los casos V y VII por no existir diferencias significativas en los rendimientos de los grupos para los distintos tipos de líderes y en la situación VI por no haber encontrado ningún grupo que se ajustara a las condiciones establecidas en las variables independientes lo que ha imposibilitado la obtención de resultados para este octante.

La tercera hipótesis de las denominadas «Tipo I» queda plenamente confirmada en la situación VIII, de más bajo nivel de favorabilidad situacional, en donde se muestran más efectivos los grupos con líderes centrados en la tarea.

TABLA II

Rendimiento medio de los grupos y experiencia del lider

\begin{tabular}{crcc}
\hline Situac. & Grupo & Rto. medio & Exp. líder (años) \\
\hline 1 & 1 & $92,51 \%$ & 19 \\
1 & 9 & $92,21 \%$ & 15 \\
2 & 2 & $91,05 \%$ & 9 \\
2 & 10 & $91,36 \%$ & 5 \\
3 & 3 & $96,37 \%$ & 14 \\
3 & 11 & $83,65 \%$ & 13 \\
4 & 4 & $82,32 \%$ & 13 \\
4 & 12 & $89,91 \%$ & 21 \\
5 & 5 & $92,51 \%$ & 13 \\
5 & 13 & $92,38 \%$ & 9 \\
7 & 7 & $84,75 \%$ & 16 \\
7 & 15 & $86,28 \%$ & 17 \\
8 & 8 & $91,40 \%$ & 16 \\
8 & 16 & $83,05 \%$ & 14 \\
\hline
\end{tabular}

En lo que se refiere al segundo grupo de hipótesis, los datos (Tabla II) nos indican que no existe diferencia significativa en el rendimiento de los distintos grupos en relación a la experiencia del lider, aunque si parece exis- 
tir una cierta correlación entre el rendimiento de éstos y la coincidencia del tipo de motivación del lider del grupo con la de los superiores jerárquicos a éste; los datos (Tabla III) muestran que cuando el tipo de motivación del líder y del superior coinciden en cualquiera de los dós sentidos (centrados hacia la tarea o hacia las relaciones), el rendimiento del grupo es mayor que si éstas difieren.

\section{TABLA III}

Rendimientos de los diferentes talleres y tipo de motivación de los lideres y sus superiores

\begin{tabular}{llll}
\hline \multicolumn{1}{c}{ Grupo } & \multicolumn{1}{c}{ Motiv. superior } & \multicolumn{1}{c}{ Motiv. lider } & Rto. medio \\
\hline \multirow{2}{*}{ Taller 1} & C. Tarea & C. Tarea & $91,40 \%$ \\
& & C. Relaciones & $83,05 \%$ \\
Taller 2 & C. Tarea & C. Tarea & $87,81 \%$ \\
& & C. Relaciones & $85,54 \%$ \\
Taller 3 & C. Relaciones & C. Tarea & $90,93 \%$ \\
& \multirow{2}{*}{ Taller 4} & C. Tarea & $91,36 \%$ \\
& C. Tarea & C. Tarea & $92,51 \%$ \\
\hline
\end{tabular}

TABLA IV

Datos de accidentabilidad según el tipo de lider

\begin{tabular}{lccccccc}
\hline \multirow{2}{*}{ Grupo } & \multicolumn{2}{c}{ N. accidentes } & & \multicolumn{2}{c}{ Horas perdidas } & \multirow{2}{*}{ I.F. } \\
\cline { 2 - 5 } & L. Tarea & L. Relac. & L. Tarea & L. Relac. & & \\
\hline Taller 1 & 2 & 7 & 192 & 512 & 28,81 & 0,19 \\
Taller 2 & 2 & - & 472 & - & 12,41 & 0,37 \\
Taller 3 & 1 & - & 832 & - & 6,20 & 0,65 \\
Taller 4 & 5 & 3 & 288 & 80 & 22,90 & 0,13 \\
\hline TOTAL & 10 & 10 & 1.784 & 592 & & \\
\hline
\end{tabular}

Por lo que respecta al nivel de accidentabilidad, los datos (Tabla IV) han resuelto que no existe una diferencia significativa entre los grupos dirigidos por un tipo de lider $\boldsymbol{u}$ otro. Se ha producido una mayor incidencia en la gravedad de los accidentes producidos en grupos con líder centrado en la tarea, lo que puede responder a la simple casuística.

\section{DISCUSION Y CONCLUSIONES}

El análisis de los datos obtenidos, en relación al marco de referencia organizacional de realización del presente estudio, nos lleva a las siguientes conclusiones:

1. La ausencia de diferencia significativa en las situaciones I y II del modelo, puede deberse a la consideración de "tarea estructurada" como aquélla en donde existe el máximo nivel de automatización y una baja tasa 
de toma de decisiones por parte del grupo. Cabe pensar que en este tipo de tareas es mayor el peso específico del factor máquina, lo que decide que sea éste y no el factor humano el que establezca las diferencias de productividad y rendimiento.

La segunda hipótesis, reflejada en las situaciones IV, V, VI y VII, queda validada para los casos IV y VII lo que confirma que los grupos en regulares condiciones de favorabilidad son más eficaces cuando su líder se encuentra centrado en las relaciones; se trata en estos dos casos de grupos no demasiado estables pero sí con un mayor nivel de intervención por parte de sus miembros, en los trabajos a realizar, que la que se producía en las situaciones I y II. Estos resultados coinciden con los obtenidos por Shaw y Blum (1966), Fiedler (1971) y Hardy (1975).

La no existencia de diferencia significativa entre la productividad de los grupos de líder centrado en las relaciones y líder centrado en la tarea para la situación $\mathrm{V}$, se justifica por las mismas razones (tarea muy estructurada) que en las situaciones I y II.

Los resultados para la tercera hipótesis (situación VIII) parecen demostrar la mayor eficacia de grupos con líder centrado en la tarea. En estos tipos de trabajo existe un mayor nivel en la toma de decisiones por parte del factor humano, tanto del líder como de los miembros del grupo, así como una inferior especificidad en las técnicas y métodos de realización de la labor, lo que se define en la misma línea de los resultados anteriores.

2. No hemos encontrado correlación entre la experiencia del líder (medida por el número de años en la categoría) y el nivel de eficacia grupal. Estos resultados se asemejan a los obtenidos por House (1973) y Dunnette y Campbell (1970) los cuales concluyeron en sus investigaciones sobre el tema exponiendo la falta de evidencia para relacionar el entrenamiento o la experiencia del líder con la eficacia en la ejecución del liderazgo. Fleishman, Harris y House (1973) y Newport (1963) llegaron a conclusiones similares.

La confirmación de la hipótesis que expone una mayor eficacia en los grupos con un líder cuyo tipo de motivación coincide con la de su superior nos lleva a pensar en la existencia de un continuo reforzamiento, por parte del superior, a las actuaciones de aquellos líderes de su mismo tipo de motivación, lo que tendería a hacer prevalecer a estos últimos en su conducta de liderazgo con más firmeza y eficacia, consiguiendo de esta manera una situación propicia al éxito de su estilo de liderazgo.

Los resultados sobre el nivel de accidentabilidad no reflejan diferencia entre unos grupos y otros, lo que en principio parece descartar la relación de estos accidentes con las posibles diferencias de presión hacia los miembros del grupo por parte de los diferentes tipos de líder, como implícitamente planteábamos en nuestra hipótesis.

La falta de coincidencia entre las predicciones de algunas de las situaciones del Modelo de Contingencia de Fiedler y los resultados obtenidos, nos hacen pensar en la necesidad de su revisión, sobre todo para su aplicación a organizaciones en continuo cambio y de tecnología avanzada, como la que hemos estudiado.

La progresiva sustitución del hombre por la máquina (informática, elec- 
trónica, automatización, etc.), que se manifiesta de forma más pronunciada en la toma de decisiones elementales sobre los medios de producción, ponen en evidencia el desarrollo de un nuevo papel del factor humano en las organizaciones de producción, lo que necesariamente debe situar los estudios de la Psicología social, en estos contextos, hacia un análisis meticuloso y detallado de las conductas de las personas en este nuevo medio, como base para una posterior revisión de las leyes que rigen los fenómenos grupales.

\section{Referencias}

Ashour, A. (1973). "The Contingency Model of leadership effectiveness: an evaluation». Organizational behavior and Human Performance, 9, 339-355.

ASHOUR, A. (1973). "Further discurssion of Fiedler's contingency model of leadership effectiveness.. Organizational behavior and Human Performance, 10, 330-350.

Campbell, J. y cols. (1970). Managerial Performance and effectiveness. McGraw-Hill, Nueva York.

FIEDLER, F. (1967). A theory of leadership effectiveness. McGraw-Hill, Nueva York.

FIEDLER, F. (1971). "Validation and extension of the Contingency Model of leadership effectiveness: A review of empirical finding». Psychological Bulletin, 76, 128-248.

FIEDLER, F. (1978). "The Contingency Model and the Dynamics of the leadership process". Advances in Experimental Social Psychology, 11, 59-112.

FleISHMAN, E. y cols. (1955). Leadership an supervision industry. University of Ohio, Bureau of Educational Research.

FleISHMAN, E. y HUNT, J. (1973). Currents developments in the studt of leadership. University of Illiniois.

GRAEN, G. y cols. (1970). "The contingency model leadership effectiveness antecedent and evidential result». Psychological Bulletin, 74, 285-296.

HARDY, R. (1975). "A test of poor leader-member relations cells of the Contingency Model on elementary school children". Child Development, 45, 958-964.

HoskING, D. (1978). A critical evaluation of Fiedler's predictor measures of leadership effectiveness. University of Warwick.

HOUSE, R. (1973). A path goal theory of leader effectiveness. En Fleishman y Hunt (comp.). University of Illinois.

KATZ, D. y KAHN, R. (1966). The social psychology of organization. Wiley, Nueva York.

LePlat, J. y CUNy, X. (1977). Psicologia del trabajo. Pablo del Río, Madrid.

Mitchel, T. y cols. (1970). "The Contingency Model: Critics and suggestions». Academy of Management Journal, 13, 253-268.

MORAlEs, J. (1985). "El liderazgo en los grupos». En C. Huici (comp.). Estructura y procesos de grupo, U.N.E.D.

NEWPORT, M. (1963). Midle management development in industrial organization. University of Illinois.

PEIRO, J. (1984). Psicologia de las Organizaciones, U.N.E.D.

RICE, R. y ChEmERS, M. (1973). "Predicting the emergence of leaders using Fiedler's Contingency Model of leadership effectiveness". Journal of Applied Psychology, 57, 281-287.

RUBIO, J. (1986). «Estudio empírico sobre la validez del Modelo de la Contingencia de Fiedlern. Revista de Psicología General y Aplicada, 41, 601-604.

SHAw, M. y BLUM, J. (1966). «Effets of leadership style upon group performance as a function of task structure*. Journal of Personality and Social Psychology, 3; 238-242.

Siguan, M. (1963). Problemas humanos del trabajo industrial. Rialp, Madrid. 